\title{
Perfil de trabalhadores da enfermagem de hospitais universitários e as cargas de trabalho: análise por cluster ${ }^{a}$
}

The profile of nursing workers at university hospitals and workloads: a cluster analysis Perfil de trabajadores de enfermería de hospitales universitarios y las cargas de trabajo: análisis por cluster

\author{
Deciane Pintanela de Carvalho ${ }^{1}$ (1) \\ Laurelize Pereira Rocha ${ }^{1}$ (D) \\ Aline Neutzling Brum² ${ }^{2}$ \\ Evilin Diniz Gutierres ${ }^{1}$ (D) \\ Aline Carniato Dalle Nogario ${ }^{1}$ (D) \\ Simoní Saraiva Bordignon ${ }^{1}(1)$
}

1. Universidade Federal de Rio Grande. Rio Grande, RS, Brasil.

2. Universidade Federal do Pampa. Dom Pedrito, RS, Brasil.
Autor correspondente:

Deciane Pintanela de Carvalho.

E-mail: deciane.carvalho@gmail.com

Recebido em 01/06/2021.

Aprovado em 21/11/2021.

DOI:https://doi.org/10.1590/2177-9465-EAN-2021-0194

\section{RESUMO}

Objetivo: analisar o perfil sociodemográfico, laboral e a intensidade das cargas de trabalho de trabalhadores da enfermagem de hospitais universitários. Método: quantitativo e transversal, com 361 trabalhadores de enfermagem, entre novembro de 2019 e fevereiro de 2020, por meio da Escala de Cargas de Trabalho nas Atividades de Enfermagem. Realizou-se análise de cluster. Resultados: formaram-se quatro clusters com trabalhadores do sexo feminino (2, 3 e 4) e sexo masculino (1). A exposição às cargas de trabalho foi identificada como intensa (1, 2 e 3 ) e pouco intensa (4). As unidades de trabalho evidenciadas com mais frequência nos clusters 1, 2 e 3 foram a Rede de Urgência e Emergência, Pediátrica e Clínica Médica, e no cluster 4, a Maternidade e Clínica Cirúrgica. Os clusters 1 e 3 identificaram com mais frequência o período de trabalho de 1 a 5 anos, no cluster 2, foi de 15 a 20 anos, e no cluster 4, inferior a um ano. Conclusão e implicações para a prática: a identificação dos quatro clusters possibilitou a análise do perfil de trabalhadores de enfermagem, permitindo o planejamento de intervenções direcionadas a minimizar as cargas de trabalho de acordo com as particularidades de cada grupo.

Palavras-chave: Análise por Conglomerados; Carga de Trabalho; Enfermagem; Hospitais Universitários; Trabalho.

\section{Abstract}

Objective: to analyze the sociodemographic and occupational profile and intensity of workloads among nursing professionals working in university hospitals. Method: This quantitative and cross-sectional study addressed 361 nursing workers from November 2019 to February 2020, using the Escala de Cargas de Trabalho nas Atividades de Enfermagem and cluster analysis. Results: Four clusters were identified: with female workers (2,3, and 4) and male workers (1). Exposure to workload was reported to be intense (1, 2, and 3) and a little intense (4). The work units most frequently identified in clusters 1, 2, and 3 were the Emergency and Urgent Care Unit, Pediatrics, and Medical Clinic, whereas Maternity Services and Surgical Clinics were the units most frequently identified in cluster 4. Most workers in clusters 1 and 3 had from 1 to 5 years of experience, cluster 2 from 15 to 20 years, and the workers in cluster 4 had less than one year of work experience. Conclusion and implications for practice: the identification of four clusters enabled analyzing the profile of the nursing workers, which favors the planning of interventions intended to minimize workloads according to the specificities of each group.

Keywords: Cluster Analysis; Workload; Nursing; Hospitals, University; Work.

\section{RESUMEN}

Objetivo: analizar el perfil sociodemográfico, laboral y la intensidad de las cargas de trabajo de trabajadores de enfermería de hospitales universitarios. Método: cuantitativo y transversal con 361 trabajadores de enfermería, entre noviembre de 2019 y febrero de 2020, por medio de la Escala de Cargas de Trabajo en las Actividades de Enfermería. Se realizó el análisis de cluster Resultados: se formaron cuatro clusters, con trabajadores del sexo femenino (2, 3 y 4) y sexo masculino (1), la exposición a las cargas de trabajo fue identificada como intensa (1, 2 y 3) y poco intensa (4). Las unidades de trabajo evidenciadas con más frecuencia en los clusters 1, 2 y 3 fueron la Unidad de Cuidados de Emergencia y Urgencia, Pediatría y Clínica Médica y en e cluster 4, los Servicios de Maternidad y de Clínica Quirúrgica. Los clusters 1 y 3 identificaron con más frecuencia el período de trabajo de 1 a 5 años, en el cluster 2 fue de 15 a 20 años y en el cluster 4, inferior a un año. Conclusión e implicaciones para la práctica: la identificación de los cuatro clusters posibilitó el análisis del perfil de los trabajadores de enfermería, permitiendo la planificación de intervenciones dirigidas a minimizar las cargas de trabajo de acuerdo con las particularidades de cada grupo. Palabras clave: Análisis por Conglomerados; Carga de Trabajo; Enfemería; Hospitales Universitarios; Trabajo. 


\section{INTRODUÇÃO}

O sistema de saúde no Brasil busca fortalecer e garantir à população acesso aos serviços. No entanto, a saúde e seus trabalhadores têm vivenciado transformações que modificaram o modelo de assistência e cuidado aos pacientes. Evidencia-se que os trabalhadores de enfermagem apresentam desgastes, adoecimentos, salários defasados e mercado de trabalho comprometido ${ }^{1}$.

A enfermagem é uma categoria profissional formada, em sua maioria, por mulheres, que estão expostas a intenso desgaste físico e psíquico. É composta por mais de dois milhões de trabalhadoras, como enfermeiras, técnicas em enfermagem e auxiliares de enfermagem, as quais atuam em diferentes estruturas organizacionais do sistema de saúde brasileiro, centros de saúde, ambulatórios, Unidades Básicas de Saúde, Unidades de Pronto Atendimento, Serviços de Atendimento Móveis de Urgência, Hospitais, entre outros².

Estudo que comparou o trabalho em quatro hospitais universitários, dois no Brasil, um na Argélia e um na França, destacou que os trabalhadores de enfermagem desempenham atividades em ambientes de trabalho com espaço físico inadequado, recursos materiais insuficientes, déficit de trabalhadores, grande rotatividade, absenteísmo e alta demanda de tarefas administrativas. Tais fatores comprometem a organização dos serviços e geram prejuízos também nas atividades de ensino ${ }^{3}$.

Esses aspectos relacionados às condições de trabalho nos remetem às cargas de trabalho, identificadas como elementos presentes no ambiente de trabalho que interatuam com os trabalhadores, gerando desgastes físicos e psíquicos. Essas cargas são caracterizadas como físicas, químicas, biológicas, mecânicas, psíquicas e fisiológicas ${ }^{4}$.

No Brasil, verificou-se que a carga de trabalho causa influência negativa na saúde dos trabalhadores. Entre as condições de trabalho que geram cargas de trabalho, estão as altas demandas físicas, adoção de posturas impróprias por movimentos repetitivos na realização das atividades, centralização de decisões e divisão de tarefas, duplo vínculo de trabalho, horas extras, ritmo de trabalho excessivo, mobiliário, materiais, espaço físico e instrumentos inadequados ${ }^{5}$.

Em consequência da presença das cargas de trabalho, ocorrem desgastes à saúde, como dor lombar, cansaço mental ${ }^{5,6}$, fadiga, mau humor, cansaço físico ${ }^{4}$, dor em membros superiores, cervical, torácica, membros inferiores e nas articulações, contratura muscular, edema em membros inferiores, falta de ar, problemas digestivos, cefaleia, nervosismo, esquecimento, insônia, irritação nos olhos, fraqueza e tontura6 .

Estudo no Rio de Janeiro, Brasil, identificou o perfil de trabalhadores de enfermagem que atuava em ambulatórios de hospitais universitários, a partir da caracterização sociodemográfica e ocupacional de 388 participantes. Evidenciou-se que $88,6 \%$ eram do sexo feminino, a faixa etária com maior frequência era de 50 anos para $48,4 \%$ dos trabalhadores. $68,3 \%$ deles possuíam ensino superior completo, 30,1\% eram enfermeiros, 50,5\% eram técnicos em enfermagem e 19,1\% eram auxiliares de enfermagem. Com relação à presença de doenças, verificou-se que 50,8\% deles referiram estresse, $46,1 \%$, doença osteomuscular, 45,4\%, varizes, $39,2 \%$, colesterol elevado, e 38,7\%, hipertensão arterial ${ }^{7}$.

Nos hospitais universitários, o trabalho desenvolvido envolve o cuidado junto ao paciente hospitalizado, atividades de gestão, e ainda ações vinculadas ao ensino e pesquisa ${ }^{8}$. Assim, consideraram-se os hospitais universitários como cenário de estudo, pois são ambientes onde os trabalhadores possuem alta demanda de trabalho voltada à atenção à saúde, gerenciamento, administração e atividades de ensino, pesquisa e extensão. Neste sentido, este estudo busca possibilitar que as instituições de saúde e universidades vinculadas tracem estratégias de prevenção a desgastes à saúde dos trabalhadores, por meio da redução das cargas de trabalho, promovendo ambientes de trabalhos saudáveis e condições de trabalho adequadas.

Desse modo, apresenta-se como questão de pesquisa: qual o perfil de trabalhadores de enfermagem de hospitais universitários, de acordo com suas características sociodemográficas e laborais e a intensidade das cargas de trabalho? Teve-se como objetivo analisar o perfil sociodemográfico, laboral e a intensidade das cargas de trabalho de trabalhadores da enfermagem de hospitais universitários.

\section{MÉTODO}

Estudo quantitativo, transversal e analítico, com trabalhadores de enfermagem, enfermeiros, técnicos e auxiliares de enfermagem, atuantes em dois hospitais universitários no Rio Grande do Sul, Brasil. Ambos os hospitais pertencem a universidades públicas, vinculados à Empresa Brasileira de Serviços Hospitalares.

Para o cálculo amostral, considerou-se a população de 752 trabalhadores de enfermagem nos dois hospitais universitários, que atuavam em unidades de assistência direta ao paciente, não sendo contabilizados os trabalhadores que desenvolviam suas atividades em setores administrativos. Utilizou-se a fórmula estatística para cálculo amostral em populações finitas, empregando-se o nível de confiança de $95 \%$ e erro amostral de 5\%, obtendo-se uma amostra mínima de 255 participantes. Utilizou-se amostragem não probabilística por conveniência ${ }^{9}$, com a finalidade de atingir o maior número de participantes.

Como critérios de inclusão, elencou-se a atuação nas seguintes unidades de assistência: Clínica Médica; Clínica Cirúrgica; Serviço de Pronto Atendimento (SPA); Rede de Urgência e Emergência (RUE); Clínica Obstétrica; Maternidade; Clínica Pediátrica; Centro Cirúrgico; Unidade de Terapia Intensiva (UTI) Geral; e UTI Neonatal. Estar de férias ou licenças de qualquer natureza no momento da coleta de dados foi critério de exclusão.

A coleta de dados ocorreu entre novembro de 2019 e fevereiro de 2020, por um instrumento semiestruturado com variáveis sociodemográficas e laborais e a Escala de Cargas de Trabalho nas Atividades de Enfermagem (ECTAE), previamente elaborada e validada com trabalhadores de enfermagem. A medida de coeficiente de confiabilidade do instrumento foi verificada por meio do cálculo do alfa de Cronbach, o qual apresentou o valor de 0,87 , comprovando sua fidedignidade.

A ECTAE foi previamente desenvolvida por meio de um guia de oito etapas ${ }^{10}$, com 22 questões afirmativas de situações 
de exposição às cargas de trabalho durante as atividades de enfermagem, de acordo com o referencial teórico ${ }^{4}$. A ECTAE apresenta seis construtos que representam as cargas de trabalho psíquicas, fisiológicas, biológicas, mecânicas, físicas e químicas (Quadro 1). Utilizaram-se duas escalas Likert de cinco pontos de intensidade (nada intensa $=0$; pouco intensa $=1$; intensa $=$ 2; muito intensa $=3$; extremamente intensa $=4$ ) e frequência (nada frequente $=0$; pouco frequente $=1$; frequente $=2$; muito frequente $=3$; extremamente frequente $=4$ ).

Para análise dos dados, iniciou-se a digitação e a organização no Microsoft Office Excel2020. Após, os dados foram transportados para realização do processamento e análise no Statistical Package for Social Sciences, versão 24. Para a caracterização dos participantes, utilizou-se estatística descritiva com distribuição de frequências absolutas e relativas.

Realizou-se a análise de cluster, identificada como um grupo de técnicas multivariadas para agrupar objetos de acordo com as características que possuem. Desta forma, devem apresentar elevada homogeneidade interna (dentro dos agrupamentos) e elevada heterogeneidade externa (entre agrupamentos). Para isso, utilizou-se o método twostep cluster ou análise de cluster de duas etapas, ferramenta exploratória que permite a análise de grandes conjuntos de dados, usando variáveis categóricas e contínuas, além de ser capaz de identificar automaticamente o número ideal de cluster ${ }^{11}$.

Empregou-se a análise de componentes principais, com a finalidade de reduzir o número de variáveis utilizadas e a medida de distância de verossimilhança, que mediu a probabilidade de distribuição sobre as variáveis. Como critério para as variáveis categóricas, testou-se a independência de duas variáveis por meio do procedimento crosstabs e o Teste Qui-Quadrado $(p=0,000)$. Já nas variáveis contínuas, verificou-se a independência entre duas variáveis através de correlações bivariadas, e o procedimento de médias foi o critério adotado no algoritmo para seleção da variável ${ }^{11}$.

Assim, identificou-se a formação de quatro grupos a partir das variáveis preditoras do perfil dos trabalhadores da enfermagem - sexo, tempo e unidade de trabalho e intensidade das cargas de trabalho -, as quais apresentaram qualidade na formação dos clusters, com valores entre 0 e 1, de acordo com a medição de coesão. Destaca-se que outras variáveis foram selecionadas na análise, mas foram excluídas pelo algoritmo, uma vez que não apresentaram heterogeneidade extragrupos e homogeneidade intragrupos.

A normalidade dos dados foi verificada por meio do Teste Kolmogorov-Smirnov ( $p=0,000)$, identificando uma distribuição normal. Portanto, utilizou-se o Teste Paramétrico Análise de Variância (ANOVA) para identificar as diferenças entre as médias dos grupos formados. Foi adotado o valor de $p<0,05$ como significância estatística para o teste ANOVA. O estudo foi aprovado pelo Comitê de Ética em Pesquisa envolvendo Seres Humanos, conforme Parecer 79/2019 e CAAE 10639819.4.0000.5324.

\section{RESULTADOS}

Participaram do estudo 361 trabalhadores de enfermagem, $295(81,7 \%)$ eram do sexo feminino e 66 (18,3\%) eram do masculino, apresentando idade média de 38,9 anos (DP $\pm 8,6$ anos). De acordo com a função de trabalho, 97 (26,9\%) eram enfermeiros, $233(64,5 \%)$ eram técnicos em enfermagem e $31(8,6 \%)$ eram auxiliares de enfermagem.

Com relação ao quantitativo de trabalhadores por unidade de trabalho, verificou-se $20(5,5 \%)$ na Clínica Obstétrica, $49(13,4 \%)$ na Maternidade, 28 (7,8\%) na Clínica Pediátrica, $33(9,1 \%)$ no SPA, 57 (15,8\%) na Clínica Médica, 31 (8,6\%) na Clínica Cirúrgica, 20 (5,5\%) no Centro Cirúrgico, 25 (6,9\%) na UTI Geral, 46 (14,4\%) na UTI Neonatal e 52 (14,4\%) na RUE.

$\mathrm{Na}$ análise de cluster das variáveis sociodemográficas e laborais de trabalhadores de enfermagem e a intensidade das cargas de trabalho, formaram-se quatro clusters com boa qualidade, conforme Figura 1.

Na Tabela 1, verificou-se a distribuição dos trabalhadores de enfermagem de acordo com os clusters formados por meio das variáveis preditoras.

Na análise dos agrupamentos, identificou-se que o cluster 1 foi formado por 66 trabalhadores de enfermagem, exclusivamente do sexo masculino (100\%), com tempo de trabalho mais frequente de 1 a 5 anos (34,8\%), e a unidade de trabalho mais frequente

Quadro 1. Descrição dos itens que compõem a ECTAE.

\begin{tabular}{|l|l|}
\hline \multicolumn{1}{|c|}{ Construto } & \multicolumn{1}{c|}{ Atividades de enfermagem } \\
\hline F1 - Cargas psíquicas & $\begin{array}{l}\text { Tarefas administrativas, assistenciais e atividades de ensino, pesquisa e extensão, comunicação, } \\
\text { orientação e supervisão, trabalho em equipe, atenção constante, violência psicológica e/ou } \\
\text { moral, tensão no trabalho. } \\
\text { Manipulação de peso, transporte de pacientes, esforço físico. }\end{array}$ \\
\hline F2 - Cargas fisiológicas & $\begin{array}{l}\text { Presença de microrganismos, secreções, fluídos corporais e realização de procedimentos. } \\
\text { F3 - Cargas biológicas }\end{array}$ \\
\hline F4 - Cargas mecânicas & $\begin{array}{l}\text { Sofrer violência física, quedas e acidentes de trabalho. } \\
\text { Condições de infraestrutura, iluminação, espaço físico, materiais, descarte de resíduos e } \\
\text { choque elétrico. } \\
\text { Freparo e administração de medicamentos e manipulação de produtos de limpeza e } \\
\text { desinfecção de materiais. }\end{array}$ \\
\hline
\end{tabular}

Fonte: elaboração própria. 
foi a RUE, com $28,8 \%$ dos trabalhadores. Nesse cluster, os trabalhadores identificaram a exposição às cargas de trabalho como intensa, com média de 2,05.

O cluster 2 correspondeu ao maior agrupamento $(n=143)$, com trabalhadores do sexo feminino (100\%), tempo e unidade de trabalho mais frequente, nesse cluster, foram de 15 a 20 anos por $25,9 \%$ delas e Unidade Pediátrica com $14 \%$ dos trabalhadores, e a exposição às cargas de trabalho foi identificada como intensa, com média de 2,08.

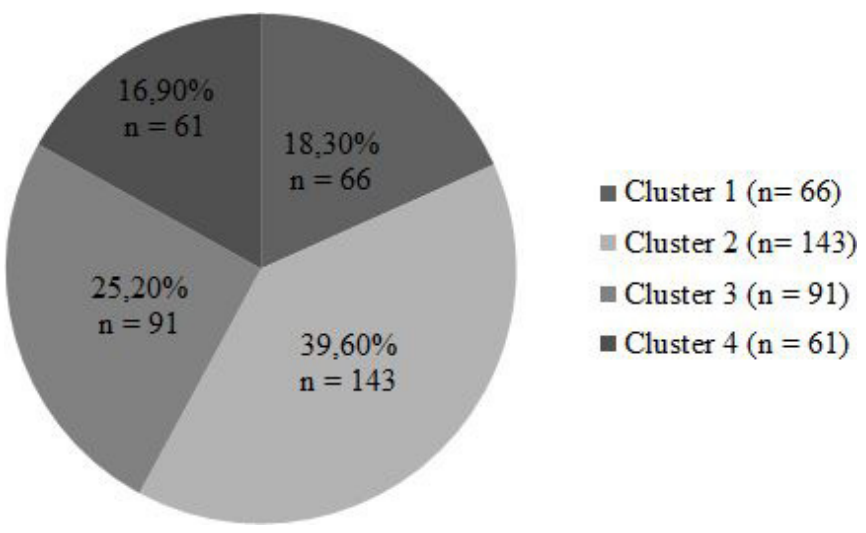

Figura 1. Tamanho dos clusters formados.
O cluster 3 foi composto por 91 trabalhadores de enfermagem, do sexo feminino (100\%), que exercem atividades de um ano a cinco anos (100\%). A unidade mais frequente foi a Clínica Médica, com 25,3\% das trabalhadoras, e a exposição às cargas de trabalho foi evidenciada como intensa, com média de 2,21, maior média dos agrupamentos.

O cluster 4 foi composto por 61 trabalhadores de enfermagem, do sexo feminino (100\%), com tempo de trabalho mais frequente de menos de 1 ano, identificado por $93,4 \%$ das trabalhadoras. As unidades mais frequentes, nesse cluster, foram a Maternidade e a Clínica Cirúrgica, com 18\% das trabalhadoras em cada, e a exposição as cargas de trabalho foi identificada como pouco intensa, com média de 1,80, diferenciando-se dos demais agrupamentos com relação à avaliação da intensidade das cargas de trabalho.

Para melhor visualização da exposição às cargas de trabalho, a Tabela 2 apresenta a intensidade das cargas de trabalho a partir das médias obtidas em cada cluster, a qual também identifica, por meio do teste ANOVA, diferença significativa entre os clusters e o instrumento, sugerindo que a média foi diferente em cada cluster, validando os clusters encontrados.

Tabela 1. Clusters de acordo com as variáveis preditoras.

\begin{tabular}{|c|c|c|c|c|c|}
\hline \multirow{2}{*}{\multicolumn{2}{|c|}{ Variáveis }} & Cluster $1(n=66)$ & Cluster $2(n=143)$ & Cluster $3(n=91)$ & Cluster $4(n=61)$ \\
\hline & & n (\%) & $\mathrm{n}(\%)$ & $\mathrm{n}(\%)$ & $\mathrm{n}(\%)$ \\
\hline \multirow{2}{*}{ Sexo } & Feminino & - & $143(100)$ & $91(100)$ & $61(100)$ \\
\hline & Masculino & 66 (100) & - & - & - \\
\hline \multirow{8}{*}{ Tempo de trabalho } & $<1$ ano & $1(1,5)$ & $2(1,4)$ & - & $57(93,4)$ \\
\hline & De 1 a 5 anos & $23(34,8)$ & $16(11,2)$ & $91(100)$ & - \\
\hline & De 5 a 10 anos & $11(16,7)$ & $31(21,7)$ & - & - \\
\hline & De 10 a 15 anos & $7(10,6)$ & $33(23,1)$ & & \\
\hline & De 15 a 20 anos & $8(12,1)$ & $37(25,9)$ & - & $2(3,3)$ \\
\hline & De 20 a 25 anos & $3(4,5)$ & $12(8,4)$ & & $1(1,6)$ \\
\hline & De 25 a 30 anos & $5(7,6)$ & $8(5,6)$ & - & $1(1,6)$ \\
\hline & $>30$ anos & $2(3,0)$ & $4(2,8)$ & - & - \\
\hline \multirow{10}{*}{ Unidade } & RUE & $19(28,8)$ & $19(13,3)$ & $13(14,3)$ & $1(1,6)$ \\
\hline & Clínica Obstétrica & - & $1(0,7)$ & $10(11)$ & $9(14,8)$ \\
\hline & Maternidade & $2(3,0)$ & $18(12,6)$ & $18(19,8)$ & $11(18)$ \\
\hline & Clínica Pediátrica & $3(4,5)$ & $20(14)$ & - & $5(8,2)$ \\
\hline & SPA & $11(16,7)$ & $15(10,5)$ & - & $7(11,5)$ \\
\hline & Clínica Médica & $13(19,7)$ & $11(7,7)$ & $23(25,3)$ & $10(16,4)$ \\
\hline & Clínica Cirúrgica & $4(6,1)$ & $10(7,0)$ & $6(6,6)$ & $11(18)$ \\
\hline & Centro Cirúrgico & $1(1,5)$ & $18(12,6)$ & - & $1(1,6)$ \\
\hline & UTI Geral & $5(7,6)$ & $12(8,4)$ & $5(5,5)$ & $3(4,9)$ \\
\hline & UTI Neonatal & $8(12,1)$ & $19(13,3)$ & $16(17,6)$ & $3(4,9)$ \\
\hline \multirow{2}{*}{\multicolumn{2}{|c|}{ Intensidade das cargas de trabalho }} & $\overline{\mathrm{x}}(\mathrm{DP})$ & $\overline{\mathrm{x}}(\mathrm{DP})$ & $\overline{\mathrm{x}}(\mathrm{DP})$ & $\overline{\mathrm{x}}(\mathrm{DP})$ \\
\hline & & $2,05(0,76)$ & $2,08(0,65)$ & $2,21(0,56)$ & $1,80(0,66)$ \\
\hline
\end{tabular}

$\overline{\mathrm{x}}$ - média; DP - desvio padrão; RUE - Rede de Urgência e Emergência; SPA - Serviço de Pronto Atendimento; UTI - Unidade de Terapia Intensiva. 
Tabela 2. Intensidade das cargas de trabalho de acordo com os clusters formados

\begin{tabular}{|c|c|c|c|c|c|c|}
\hline Cluster & Nada intenso & Pouco Intenso & Intenso & Muito intenso & $\begin{array}{c}\text { Extremamente } \\
\text { intenso }\end{array}$ & $p$-value \\
\hline & $\bar{x}$ & $\overline{\mathrm{x}}$ & $\bar{x}$ & $\bar{x}$ & $\overline{\mathrm{x}}$ & \\
\hline 1 & - & - & 2,05 & - & - & \\
\hline 2 & - & - & 2,08 & - & - & $0,002 *$ \\
\hline 3 & - & - & 2,21 & - & - & \\
\hline 4 & - & 1,80 & - & - & - & \\
\hline
\end{tabular}

$\overline{\mathrm{x}}$ - média; ${ }^{*}$ - $\mathrm{p}<0,05$

\section{DISCUSSÃO}

Dos quatro clusters formandos, três deles (2, 3 e 4) são compostos por trabalhadoras do sexo feminino, e um (1), do sexo masculino. A exposição às cargas de trabalho foi identificada como intensa nos clusters 1, 2 e 3, e como pouco intensa no cluster 4. As unidades de trabalho identificadas com mais frequência nos clusters 1, 2 e 3 foram, respectivamente, a RUE, a Pediátrica e a Clínica Médica, e no cluster 4, foram a Maternidade e a Clínica Cirúrgica. Os clusters 1 e 3 identificaram com mais frequência o período de trabalho de 1 a 5 anos, no cluster 2 , o tempo de 15 a 20 anos, e no cluster 4 , o período inferior a um ano.

Quanto à predominância de clusters formados com trabalhadores do sexo feminino, evidencia-se que este resultado é semelhante ao encontrado em estudo com 265 enfermeiras e 810 técnicas e auxiliares de enfermagem de hospitais públicos, sendo $90,1 \%$ e $86,9 \%$ do sexo feminino, respectivamente ${ }^{12}$. A enfermagem tem como característica ser composta por uma equipe predominantemente feminina, conforme evidencia o Perfil da Enfermagem no Brasil, que identifica 85,1\% de trabalhadoras. Entretanto, aponta um aumento crescente de trabalhadores do sexo masculino $(14,4 \%)$ desde o início da década de $1990^{13}$.

De acordo com isso, o cluster 1 foi agrupado com trabalhadores do sexo masculino $(n=66)$, representando $18,3 \%$ do total da amostra. Estudos apresentam essas modificações no perfil de egressos dos cursos de enfermagem. Na Escola de Enfermagem da Universidade de São Paulo, no período de 1950 a 1990, o quantitativo de homens na enfermagem era de $2,37 \%$ para $97,63 \%$ de egressos do sexo feminino ${ }^{14}$. No período de 2006 a 2012, o quantitativo alterou para $7,6 \%$ do sexo masculino e $92,4 \%$ do sexo feminino ${ }^{15}$. Resultados semelhantes foram encontrados na Faculdade de Enfermagem da Universidade Federal de Juiz de Fora, que apresentou $12 \%$ de egressos do sexo masculino entre os anos de 2005 e $2017^{16}$.

Ainda no cluster 1 , a exposição as cargas de trabalho foram identificadas como intensas. Esse resultado pode estar relacionado ao fato de que o agrupamento é constituído por trabalhadores do sexo masculino e os homens experienciam desafios negativos frente ao exercício da profissão, desde sua inserção no mercado de trabalho ${ }^{17}$. Um exemplo disso é a atuação do enfermeiro na assistência à saúde da mulher, evidencia-se que mulheres que realizam consulta ginecológica, em sua maioria preferem que os atendimentos sejam realizados por enfermeiras ${ }^{18}$. Além da assistência, os enfermeiros tendem a ocupar com frequência cargos de liderança e docência ${ }^{19}$, atividades que também podem aumentar a exposição as cargas de trabalho.

Já os agrupamentos formados exclusivamente por trabalhadoras do sexo feminino caracterizaram a exposição às cargas de trabalho como intensa (clusters 2 e 3) e pouco intensa (cluster 4). A identificação das cargas de trabalho como presentes no ambiente laboral da enfermagem de um hospital universitário são evidenciados por outro estudo com a participação predominante de trabalhadoras do sexo feminino, destacandose as cargas biológicas, psíquicas, cargas fisiológicas, cargas químicas, cargas físicas e cargas mecânicas. Identificam-se como cargas de trabalho, a presença de vírus e bactérias no ambiente de trabalho, umidade, ocorrência de acidentes com perfurocortantes, tensão prolongada, conflitos, relacionamentos interpessoais difíceis, trabalho em turnos, realização de esforço físico e percorrer longas distâncias dentro da instituição ${ }^{6}$.

Para compreender a exposição às cargas de trabalho, também é necessário conhecer as condições de trabalho vivenciadas pelos trabalhadores nos ambientes laborais. Analisando o contexto socioambiental dos hospitais universitários e a presença de docentes e estudantes nas unidades de internação, supõe-se o aumento à exposição dos trabalhadores de enfermagem às cargas de trabalho. A universidade está ligada ao hospital, ocorrendo a articulação das atividades de assistência aos pacientes, ensino, pesquisa e extensão por meio das atividades realizadas durante o ano letivo. Assim, as relações interpessoais e interações positivas entre estudantes e trabalhadores, por exemplo, são essenciais para a manutenção de um ambiente de trabalho saudável, troca de experiências e qualidade na assistência ${ }^{20}$.

Dentre as unidades de trabalho em que os trabalhadores de enfermagem desempenham as atividades de assistência, aquelas identificadas com mais frequência nos clusters 1, 2 e 3 foram, respectivamente, a RUE, a Pediátrica e a Clínica Médica. Nessas unidades, os trabalhadores de enfermagem apresentam altos índice de perda de produtividade decorrente do adoecimento dos mesmos devido à presença das cargas de trabalho ${ }^{21}$.

O serviço de emergência é identificado como a principal porta de entrada dos pacientes no ambiente hospitalar. Desta forma, podem apresentar problemas relacionados à alta demanda de atendimento e à grande rotatividade de pacientes, características responsáveis por gerar estresse entre os trabalhadores. Dentre os itens que merecem atenção e melhorias para o fortalecimento da assistência nesses serviços, estão a liberdade para a resolução de problemas da unidade e o apoio das instituições de saúde ${ }^{22}$. 
A Clínica Pediátrica é caracterizada por apresentar fatores no ambiente de trabalho que podem ser capazes de gerar insatisfação entre os trabalhadores como: dificuldades do trabalho em equipe e com as relações multiprofissionais; dificuldades com serviços de apoio; falta de tempo para discutir a assistência realizada; pouco apoio da instituição; baixa autonomia e dimensionamento inadequado de pessoal ${ }^{23}$. Além disso, os trabalhadores de enfermagem que atuam em pediatria referem que o trabalho neste ambiente exige um perfil diferenciado, devido à especificidade de atender à criança e sua família, sendo necessário sensibilidade e envolvimento emocional. O processo de trabalho envolve englobar o familiar nas ações de cuidado e valorizar sua participação ${ }^{24}$.

A Unidade de Clínica Médica também é caracterizada por apresentar alta complexidade e risco de morte dos pacientes. Tal fato requer a administração de medicamentos potencialmente perigosos, realização de procedimentos invasivos, além de monitorização constante, necessitando um número elevado de trabalhadores. Com isso, verificam-se relações indiretas entre o trabalho desenvolvido e o adoecimento dos trabalhadores, levando a consequências negativas na qualidade de vida e absenteísmo no ambiente hospitalar ${ }^{25}$

No cluster 4, os trabalhadores desenvolviam o trabalho com mais frequência nas unidades de Maternidade e Clínica Cirúrgica. A atuação em Maternidades é identificada por enfermeiros como satisfatória, uma vez que possuem autonomia na realização da assistência aos pacientes, apoio das chefias e controle sobre o que ocorre na unidade. As relações da equipe de enfermagem são estabelecidas com confiança, sobretudo acerca do que ocorre dentro do setor, utilizando como estratégia reuniões para a resolução de problemas ${ }^{26}$. Já com relação ao desenvolvimento da assistência em Clínica Cirúrgica, identifica-se que atitudes como boa colaboração e comunicação interdisciplinar, apoio de colegas, bom trabalho em equipe e oportunidade de expressar opiniões são fatores que reduzem as cargas de trabalho ${ }^{27}$.

De acordo com o tempo de trabalho identificado com maior frequência nos clusters formados, o período de 1 a 5 anos foi evidenciado por $100 \%$ dos trabalhadores do cluster 3 e por $34,8 \%$ dos trabalhadores do cluster 1 . Corroborando isso, estudo com 301 trabalhadores de enfermagem, com tempo de atuação de 1 a 4 anos para 36,5\% da amostra, evidenciou que as condições e organização do trabalho potencializam as cargas de trabalho. Fatores, como ritmo de trabalho, duração da jornada, necessidade de produtividade e cumprimento de prazos, capacidade do ambiente físico, equipamentos e instrumentos utilizados na assistência, podem ser responsáveis por gerar desgastes físicos e mentais ${ }^{5}$.

O tempo de trabalho de 15 a 20 anos foi identificado por $25,9 \%$ dos trabalhadores do cluster 2 . Outro estudo verificou a maior média de fadiga física e cognitiva entre trabalhadoras auxiliares de enfermagem que atuavam de cinco a 16 anos, e absenteísmo, com período de 11 a 29 dias de falta ao trabalho. Dessa forma, considerou o cansaço das atividades do trabalho como um possível fator de risco para o absenteísmo ${ }^{28}$.

O cluster 4 foi formado com maior frequência por trabalhadores que atuam por período inferior a um ano, representando $93,4 \%$ dos trabalhadores. A inserção recente no ambiente de trabalho pode fazer com que os trabalhadores identifiquem com menor intensidade a existência das cargas de trabalho e as consequências para a sua saúde. Isso pode ocorrer, pois enfermeiros jovens, na prática, necessitam adquirir competência técnica e funcional durante o processo de trabalho assistencial ${ }^{19}$. Apesar da presença das cargas de trabalho no ambiente laboral de hospitais públicos, muitos trabalhadores não possuem consciência dessa exposição ${ }^{5}$.

\section{CONCLUSÃO E IMPLICAÇÕES PARA A PRÁTICA}

A identificação dos quatro clusters permitiu a análise do perfil de trabalhadores de enfermagem de dois hospitais universitários localizados no Rio Grande do Sul, Brasil, de acordo com o sexo, tempo de trabalho, local de trabalho e intensidade das cargas de trabalho.

Os resultados possibilitam o planejamento de intervenções direcionadas às particularidades de cada grupo, visando diminuir as cargas de trabalho em hospitais universitários, principalmente entre mulheres na enfermagem. O conhecimento acerca das condições de trabalho, como o tempo e unidades em que são desenvolvidas as atividades de assistência, e que foram identificadas com mais frequência entre os trabalhadores de enfermagem, permitem buscar mudanças para minimizar as consequências relacionadas à presença das cargas de trabalho nesses ambientes laborais, como adoecimentos, acidentes de trabalho e afastamentos do trabalho.

A intensidade como as cargas de trabalho foram identificadas fomenta reflexões acerca das condições de trabalho em que são desenvolvidas as atividades de enfermagem, sugerindo-se mudanças que minimizem a exposição às cargas de trabalho, como melhorias relacionadas às relações interpessoais entre as equipes de trabalho, disponibilidade de infraestrutura, materiais e equipamentos em qualidade e quantidade adequadas, assim como remuneração, quantitativo de trabalhadores e carga horária compatíveis com as necessidades das instituições e trabalhadores.

Como limitação do estudo, destaca-se o desenho transversal, que impossibilita a inferência causal dos resultados obtidos. Sugere-se a realização de novas pesquisas com a finalidade de discutir os achados deste estudo com relação ao perfil dos trabalhadores da enfermagem e intensidade das cargas de trabalho.

\section{CONTRIBUIÇÕES DOS AUTORES}

Desenho do estudo. Deciane Pintanela de Carvalho. Laurelize Pereira Rocha. Aline Neutzling Brum.

Coleta ou produção dos dados. Deciane Pintanela de Carvalho. Análise de dados. Deciane Pintanela de Carvalho. Laurelize Pereira Rocha. Aline Neutzling Brum.

Interpretação dos resultados. Deciane Pintanela de Carvalho. Laurelize Pereira Rocha. Aline Neutzling Brum. Evilin Diniz Gutierres. Aline Carniato Dalle Nogario. Simoní Saraiva Bordignon.

Redação e revisão crítica do manuscrito. Deciane Pintanela de Carvalho. Laurelize Pereira Rocha. Aline Neutzling Brum. 
Evilin Diniz Gutierres. Aline Carniato Dalle Nogario. Simoní Saraiva Bordignon.

Aprovação da versão final do artigo. Deciane Pintanela de Carvalho. Laurelize Pereira Rocha. Aline Neutzling Brum. Evilin Diniz Gutierres. Aline Carniato Dalle Nogario. Simoní Saraiva Bordignon.

Responsabilidade por todos os aspectos do conteúdo e a integridade do artigo publicado. Deciane Pintanela de Carvalho. Laurelize Pereira Rocha. Aline Neutzling Brum. Evilin Diniz Gutierres. Aline Carniato Dalle Nogario. Simoní Saraiva Bordignon.

\section{EDITOR ASSOCIADO}

Gerson Luiz Marinho (D)

\section{EDITOR CIENTÍFICO}

\author{
Ivone Evangelista Cabral (B)
}

\section{REFERÊNCIAS}

1. Machado MH, Frota MA, Wermelinger MCMW, Ximenes FR No, Freire NP. Health and Nursing Systems: the national and international contexto. Cien Saude Colet. 2020;2020(1):4-5. http://dx.doi.org/10.1590/141381232020251.28562019. PMid:31859849.

2. Silva MCN, Machado MH. Health and work system: challenges for the nursing in Brazil. Cien Saude Colet. 2020;25(1):7-13. http://dx.doi. org/10.1590/1413-81232020251.27572019. PMid:31859850.

3. Scherer MDA, Conill EM, Jean R, Taleb A, Belbcke FL, Pires DEP et al. Challenges for work in healthcare: comparative study on University Hospitals in Algeria, Brazil and France. Cien Saude Colet. 2018;23(7):2265-76. http://dx.doi.org/10.1590/1413-81232018237.08762018. PMid:30020380.

4. Laurell AC, Noriega M. Processo de produção e saúde: trabalho e desgaste operário. São Paulo: Hucitec; 1989.

5. Cargnin ZA, Schneider DG, Vargas MAO, Machado RR. Non-specific low back pain and its relation to the nursing work process. Rev Lat Am Enfermagem. 2019;27:e3172. http://dx.doi.org/10.1590/15188345.2915.3172. PMid:31596408.

6. Carvalho DP, Rocha LP, Pinho EC, Tomaschewski-Barlem JG, Barlem ELD, Goulart LS. Workloads and burnout of nursing workers. Rev Bras Enferm. 2019;72(6):1435-41. http://dx.doi.org/10.1590/0034-71672017-0659. PMid:31644727.

7. Santos KM, Tracera GMP, Zeitoune RCG, Sousa KHJF, Nascimento FPB. Profile of the nursing team of university outpatient units: worker health considerations. Esc Anna Nery. 2020;24(2):1-9. http://dx.doi. org/10.1590/2177-9465-EAN-2019-0192.

8. Jacondino MB, Thofehrn MB, Amestoy SC, Moura PMM, Porto AR, Borges LR. Representations of nurses in a teaching hospital about the object, purpose and instruments of their work. Rev Fun Care Online.2019;11(1):216-21. http://dx.doi.org/10.9789/2175-5361.2019. v11i1.216-221.

9. Hill MM, Hill A. Investigação por questionário. Lisboa: Editora Sílabo; 2002.

10. Devellis RF. Scale development: theory and applications. Newbury Park: Sage; 2016

11. Hair JF, Black WC, Babin BJ, Anderson RE. Multivariate date analysis. 7th ed. Edinburgh Gate: Pearson, 2014. $739 p$.

12. Santos TA, Santos HS, Sampaio ES, Melo CMM, Souza EA, Pires CGS. Intensity of nursing work in public hospitals. Rev Lat Am Enfermagem. 2020;28:e3267. http://dx.doi.org/10.1590/1518-8345.3221.3267. PMid:32401902.
13. Machado MH, Aguiar W Fo, Lacerda WF, Oliveira E, Lemos W, Wermelinger $\mathrm{M}$ et al. Características gerais da enfermagem: o perfi sócio demográfico. Enferm Enferm Foco. 2016;7(ESP):9-14. http:// dx.doi.org/10.21675/2357-707X.2016.v7.nESP.686.

14. Costa KS, Freitas GF, Hagopian EM. Men in nursing: academic education after graduation and professional trajectory. J Nurs UFPE on line. 2017;11(3):1216-26. http://dx.doi.org/10.5205/reuol.10544-93905-1RV.1103201712.

15. Püschel VAA, Costa D, Reis PP, Oliveira LB, Carbogim FC. Nurses in the labor market: professional insertion, competencies and skills. Rev Bras Enferm. 2017;70(6):1220-6. http://dx.doi.org/10.1590/0034-71672016-0061. PMid:29160483.

16. Barbosa ACS, Luiz FS, Friedrich DBC, PüschelVAA, Farah BF, Carbogim FC. Profile of nursing graduates: competencies and professional insertion. Rev Lat Am Enfermagem. 2019;27:e3205. http://dx.doi. org/10.1590/1518-8345.3222.3205. PMid:31664413.

17. Mahadeen A, Abushaikha L, Habashneh S. Educational experiences of undergraduate male nursing students: a focus group study. Open J Nurs. 2017;7(1):50-7. http://dx.doi.org/10.4236/ojn.2017.71005.

18. Nogueira LA, Evangelista RL, Araújo CRC, Teixeira SES. Desafios da inserção do enfermeiro na assistência à saúde da mulher. Sanare [Internet]. 2017; [citado 2021 jun 1];16(1):32-8. Disponível em: https:// sanare.emnuvens.com.br/sanare/article/view/1091

19. Messias M, Gonçalves GCC, Lecca CGG, Ciampone MHT, Mira VL. Identification of the anchors of career of nurses. Rev Enferm Cent.-Oeste Min. 2017;7:e1104. http://dx.doi.org/10.19175/recom.v7i0.1104.

20. Thofehrn MB, Joner LR, Porto AR, Borel MGC, Jacondino MB, Viana NCC. Processo de trabalho: avaliação dos enfermeiros de um hospital de ensino. R Enferm UFJF. 2019;5(1):1-16. http://dx.doi.org/10.34019/24465739.2019.v5.27175

21. Carvalho DP, Rocha LP, Tomaschewski-Barlem JG, Barlem ELD, Cecagno D, Dalmolin GL. Productivity versus workloads in the nursing working environment. Rev Esc Enferm USP. 2017;51:e03301. http:// dx.doi.org/10.1590/s1980-220x2017028903301. PMid:29562050.

22. Maurício LFS, Okuno MFP, Campanharo CRV, Lopes MCBT, Belasco AGS, Batista REA. Professional nursing practice in critical units: assessment of work environment characteristics. Rev Lat Am Enfermagem. 2017;25(0):e2854. http://dx.doi.org/10.1590/1518-8345.1424.2854 PMid:28301034.

23. Costa NN, Silva AEBC, Lima JC, Bezerra ALQ, Sagawa MR, Sousa MRG. Pediatric work environment: satisfaction of nursing professionals Rev Enferm UFPE on line. 2018;12(12):3327-36. http://dx.doi. org/10.5205/1981-8963-v12i12a234975p3327-3336-2018.

24. Ribeiro JP, Gomes GC, Thofehrn MB, Porto AR, Rodrigues LP. Pediatric environment: aspects helping the work process and health production. Rev Enferm UFPE on line. 2017;11(Supl. 12):5275-81. http://dx.doi. org/10.5205/1981-8963-v11i12a22786p5275-5281-2017.

25. Pimenta CJL, Vicente MC, Ferreira GRS, Frazão MCLO, Costa TF, Costa KNFM. Health conditions and Nurses' work characteristics at a university hospital. Rev Rene. 2020;21:e43108. http://dx.doi.org/10.15253/21756783.20202143108.

26. Santos JLG, Copelli FHS, Oliveira RJT, Magalhães ALP, Gregório VRP, Endermann AL. Nursing work environment in the maternal-child nursing department at a university hospital. Rev Enferm Cent.-Oeste Min. 2018;8:e2099. http://dx.doi.org/10.19175/recom.v7i0.2099.

27. van Bogaert $P$, Peremans $L$, van Heusden $D$, Verspuy $M$, Kureckova $V$, van de Cruys $Z$ et al. Predictors of burnout, work engagement and nurse reported job outcomes and quality of care: a mixed method study. BMC Nurs. 2017;16:5. http://dx.doi.org/10.1186/s12912-016-0200-4 PMid:28115912.

28. Martinez CL, Moraga SP, Paredes CS, Vásquez AS, Villarroel CMV Occupational fatigue and work absenteeism in female assistant nurses of a high-complexity hospital, Chile. Cienc Saude Coletiva. 2020;25(1):243-9. http://dx.doi.org/10.1590/1413-81232020251.

\footnotetext{
a Extraído da tese: "Elaboração e validação do instrumento "Escala de Cargas de Trabalho nas Atividades de Enfermagem" e a relação com o presenteísmo no contexto socioambiental de hospitais universitários", de autoria de Deciane Pintanela de Carvalho, Orientação de Laurelize Pereira Rocha e Coorientação de Aline Neutzling Brum, no Programa de Pós-Graduação em Enfermagem, Universidade Federal do Rio Grande, 2020.
} 